Revue d'histoire de l'Amérique française

ZRS REVUE D.HISTOIRE DE L'AMÉRIQUE FRANÇAISE

\title{
Projets de préfectures apostoliques pour le Nord-Est du Canada (suite)
}

\section{Gaston Carrière}

Volume 17, numéro 3, décembre 1963

URI : https://id.erudit.org/iderudit/302291ar

DOI : https://doi.org/10.7202/302291ar

Aller au sommaire du numéro

Éditeur(s)

Institut d'histoire de l'Amérique française

ISSN

0035-2357 (imprimé)

1492-1383 (numérique)

Découvrir la revue

Citer cet article

Carrière, G. (1963). Projets de préfectures apostoliques pour le Nord-Est du Canada (suite). Revue d'histoire de l'Amérique française, 17(3), 396-423.

https://doi.org/10.7202/302291ar d'utilisation que vous pouvez consulter en ligne.

https://apropos.erudit.org/fr/usagers/politique-dutilisation/ 


\title{
PROJETS DE PRÉFECTURES APOSTOLIQUES POUR LE NORD-EST DU CANADA * (suite)
}

\begin{abstract}
Le mémoire, daté du 10 février 1871, est intitulé Rapport sur l'érection de deux préfectures apostoliques au Nord du Canada ${ }^{30}$.

T.R.Père - Je vous envoie un peu tardivement le rapport que vous nous avez demandé à l'occasion

Etat question

Questions préliminaires d'une décision de la S.C. de la Propagande. Conformément à cette décision les missionnaires oblats seraient chargés de former deux préfectures apostoliques en faveur des populations sauvages que depuis quelques années ils ont évangélisées sur le territoire du Canada et dans les contrées adjacentes; l'une des préfectures comprendrait les pays de l'Est et l'autre ceux de l'Ouest. Ensuite, T.R.P. vous exprimez votre volonté que ce soit la province du Canada qui prenne la responsabilité de cette entreprise. En consécuence vous nous chargez de répondre à trois questions différentes. $1^{\circ}$ Quel nom donner aux nouvelles préfectures. $2^{\circ}$ quelles limites faut-il leur assigner. $3^{\circ}$ Quels titulaires peuvent être proposés à la Sacrée Cong.

Avant de répondre à ces questions permettez, T.R.P., d'en examiner deux autres afin d'éclairer les premières et de faire constater ce que nous pouvons faire. Je me demanderai donc 1. Quel but nous devons nous proposer en formant les préfectures pour accomplir les vues de la propagande et pour assurer le plus grand bien des âmes sans dépasser les ressources dont nous disposons. 2. Quels sont les moyens dont nous disposons pour entreprendre cette œuvre.
\end{abstract}

* Voir notre Revue, XVII: 185-212.

30 Archives générales O.M.I., dossier Vandenberghe. 
1 ère question

Préfecture de l'Est Le Labrador et le territoire de la Baie d'Hudson

Beaucoup de protestants Impossible d'y avoir des établissements
1. Q. Quel est le but des nouvelles préfectures. Le sens de cette question est celui-ci: les préfectures apostoliques à organiser doivent-elles se restreindre aux missions sauvages, ou bien faut-il les étendre encore aux pays mixtes, c.a.d. à ces contrées où des établissements civilisés se mêlent aux stations sauvages et où la colonisation commence à progresser. Quelques explications sont nécessaires pour faire comprendre l'état de la question dans chacune des préfectures.

$1^{\circ}$ Préfecture de l'Est. En restreignant cette préfecture au pays purement sauvage elle ne peut comprendre que les pays qui sont situés en dehors du Canada, savoir le Labrador proprement dit, qui civilement et religieusement est une dépendance de l'île de Terreneuve. Ensuite la partie du territoire de la Baie d'Hudson qui est à l'Ouest du Labrador et qui est baignée par la mer d'Hudson. Peut-être serait-il possible d'y ajouter les îles arctiques, sur lesquelles je ne possède aucun renseignement, mais où je crois impossible de former jamais un établissement quelconque. Quant à la grande presqu'île placée entre la mer d'Hudson et le grand Océan, elle renferme pas plus de 1500 à 2000 âmes, dont les seuls Naskapis convertis par le p. Babel au nombre de 400 à 500 sont catholiques tout le reste est protestant ou infidèle. Le pays est inaccessible pendant la majeure partie de l'année et est en dehors de toute communication avec le monde civilisé. Ce pays soumis jusqu'ici à l'hon. Comp. de la B.H. offre peu de ressources pour des établissements: les parties les plus favorisées comme le district de Rupert et plusieurs postes sur les côtes de l'Océan sont occupés par les Protestants. Partout ailleurs l'hon. Comp. malgré ses ressources n'a pas réussi à former des établissements stables. Tous les rapports du p. Babel concluent à n'entretenir dans ce pays que des missionnaires ambulants, qui visitent les tribus sauvages une fois l'année.

Si la préfecture doit s'étendre aux côtes du golfe St Laurent qui fait partie du diocèse de Rimouski et qui s'étendent depuis la rivière Portneuf jusqu'au Grand Sablon où le Canada se sépare 
Préfecture de l'Ouest

3 à 4000
âmes du Labrador, c'est une étendue de côtes de 230 lieues sur une profondeur vers l'intérieur de 80 lieues. La population y est de 2000 sauvages au moins et de 2000 blancs au plus. L'on comprend que l'évêque de Rimouski désire fortement se décharger de ces missions qui sont fort coûteuses et en hiver il n'y a aucun moyen de communiquer avec ses prêtres et même en été il en est séparé par un bras de mer de 16 lieues. Actuellement quatre postes sont occupés par des prêtres: $1^{\circ}$ Celui de Bethsiamit qui est desservi par nos pères s'étend depuis Portneuf jusqu'à 20 lieues plus bas que la résidence. L'on ny compte que 33 familles canadiennes, mais nos pères ont la charge de voir les sauvages sur toute l'étendue de la côte: chaque été ils passent par les divers postes et donnent la mission aux sauvages, dont les autres prêtres ne comprennent pas la langue. En tout comme il a été dit, les sauvages sont 2000 et généralement bons chrétiens. $2^{\circ}$ Le poste de la Rivière Moisie où il y a 300 à 400 Canadiens, mais probablement ce poste ne durera pas. L'on y exploite des mines de fer. Le prêtre a été demandé par les directeurs de l'usine, mais il n'y a ni église ni maison: même, le prêtre n'a pas été rémunéré et l'évêque est sur le point de le retirer. $3^{\circ} L a$ pointe aux Esquimaux est un poste assez nombreux de pêcheurs Acadiens. Il y a plusieurs maisons. La mission se suffit à peu près. $4^{\circ}$ Le poste de Nataskuan est mêlé de sauvages et de pêcheurs canadiens. Le missionnaire y est soutenu par l'évêque de Rimouski. $5^{\circ}$ Il est question d'un autre poste où la présence du prêtre serait nécessaire, c'est le Grand Sablon et non loin de là se trouve une tribu sauvage dite des Pieds noirs, elle désire se faire chrétienne. ...

$2^{\circ}$ Préfecture de l'Ouest. En la bornant aux missions purement sauvages elle s'étendra sur tout le district du territoire de la Baie d'Hudson qui se trouve entre la préfecture de l'Est et le diocèse de St Boniface dont les limites n'ont pas été bien déterminées. L'on y compte une population de 3000 à 4000 âmes dont 1500 catholiques. Les missionnaires n'y ont encore aucune résidence. Chaque été ils visitent les postes qui sont à proximité de la baie James et dans lesquels il y a une chapelle; jusqu'ici 
ils ne se sont pas occupés des pays qui s'étendent vers l'Ouest.

jusqu'à

Témiska-

mingue

même plus bas

Mattawa centre des chantiers

Personnel nécessaire

Il faudrait au moins six sujets pour la préfecture de l'Est et deux de plus pour la plus pour de l'Ouest
La préfecture devrait naturellement comprendre aussi une partie du Canada dans la vallée de l'Ottawa depuis sa source jusqu'au lac Temiskaming inclusivement. C'est dans cette partie que se trouve la résidence des missionnaires. D'ailleurs la population y est encore sauvage puisque ces sauvages se mêlent beaucoup aux Canadiens, qui commencent à y faire quelques établissements: les chantiers aussi s'y multiplient. La population au plus y est de 800 âmes, ils dépendent des pères de Temiskaming. Il y a des chapelles en deux autres endroits. Si l'on veut reculer les limites de la Préfecture jusqu'aux dernières paroisses du diocèse d'Ottawa on les terminera à 50 lieues plus bas que Temiskaming. Là est le centre des chantiers, il y a peu de sauvages. L'on y trouve deux localités qui devront avoir un prêtre résident avant longtemps. Un endroit nommé Mattawan devient très important et sera probablement une place de progrès rapide. Jusqu'ici nos missionnaires sont les seuls prêtres qui ont exercé le ministère dans ces contrées.

2. De quels moyens peut-on disposer?

Cette question se rapporte au personnel qui est nécessaire pour desservir les préfectures et aussi aux ressources pécuniaires.

$1^{\circ}$ Personnel. Actuellement les missionnaires de Betsiamits font le travail à deux, mais évidemment nous aurions besoin de fortifier ce personnel ne fût-ce que pour avoir des remplaçants en cas d'accident. A Temiskaming les missionnaires sont quatre pendant l'hiver ils soignent les chantiers et en été ils visitent les missions sauvages. Pour la préfecture de l'Est si la Cong. se charge de la desserte du golfe, il y faudra au moins six sujets. (Pour la préfecture de l'Ouest, il faudrait immédiatement donner deux sujets de plus aux pères de Témiskaming et augmenter ensuite à mesure que des missions nouvelles se fondent.) Ainsi pour former les deux préfectures, c'est un personnel de 6 à 8 prêtres qu'il faut trouver aussitôt. 
Ressources

sont insuffisantes
Préfecture de l'Est

Les limites

$\mathrm{Ne}$ pas inclure les côtes du golfe $2^{\circ}$ Ressources pécuniaires. Actuellement les pères de Bethsiamits reçoivent un secours de 500 dollars ou mieux de $400 \$$ du gouvernement (l'évêque de Rimouski retient $50 \$$ ). En outre la propagation de la foi à Québec donne $600 \$$ pour la mission du Labrador. En tout $1050 \$$. Cette somme serait insuffisante pour prendre le service des côtes et fonder un établissement au Labrador. A Temiskaming la mission reçoit un secours de $1000 \$$ dont 800 de Québec et 200 de Montréal. Ils suppléent au reste par l'œuvre des chantiers. Dans l'organisation des préfectures ces secours nous seront retirés au bout de 3 ans. C'est donc une somme de $1600 \$$ que la Cong. devra se procurer ailleurs, ou à la Propagation de la foi en France, ou par d'autres moyens: les évêques utilisent les fonds pour leurs œuvres diocésaines. Encore pendant 3 ans nous n'aurions un secours que dans la supposition que les Evêques veulent maintenir la souscription faite à Toronto, mais déjà l'évêque de Montréal qui a souscrit pour $800 \$$ écrit ne pouvoir garantir cette somme. Cette souscription s'élevait à $2000 \$$ et était promise pour 3 ans. Ajoutez encore que cette somme de $1600 \$$ suffisant dans les conditions actuelles sera tout à fait insuffisante pour nous charger de tous les postes du Golfe. Comment aussi entreprendre des missions nouvelles. Notez qu'au lieu d'entretenir six prêtres il s'agit de pourvoir aux besoins de 12 au moins.

Après ces réflexions préliminaires j'essaierai, mon T.R.P. de répondre aux questions de la Sacrée Propagande. J'y répondrai pour chaque préfecture séparément.

\section{Préfecture du Labrador}

$1^{\circ}$ Nom. Je propose de désigner la préfecture apostolique qui doit être formée à l'est du Canada sous le nom de préfecture de Labrador. Ce nom lui même en indique bien la position.

$2^{\circ}$ Limites - Le conseil de la province est unanime pour exclure de cette préfecture les côtes du golfe St Laurent. 1. P.c.q. nous ne pouvons pas obliger nos sujets à vivre isolément. 2. La Cong. peut toujours continuer ses soins aux populations 
elles appartiennent au diocèse de Rimouski

Titulaires

Préfecture de l'ovest

Ses limites sauvages comme elle le fait maintenant. 3. Un préfet apostolique n'aura aucun moyen d'attirer des prêtres séculiers pour occuper ces postes. 4. Les moyens de soutenir ces postes font absolument défaut. Je comprends les motifs qui déterminent Mgr l'Ev. de Rimouski de se décharger de cette partie de son diocèse; cependant telles ne paraissent pas être les vues du défunt archevêque de Québec, qui croyait, si je ne me trompe pas que les côtes du golfe pourraient faire partie d'un diocèse régulier qui serait créé au Saguenay.

$3^{\circ}$ Titulaires. Nous avons trois pères aui connaissent les missions de ces pays, les RR.PP. xxx. Evidemment le premier est d'un âge trop avancé. Le choix donc doit se faire entre les deux derniers. Le Conseil unanimement a opté pour x comme étant plus conciliant et plus sociable que son compagnon, dont le dévouement tout personnel ne peut s'harmonier ni avec celui de ses frères, ni avec les exigences extérieures.

\section{Préfecture de la Baie d'Hudson}

$1^{\circ}$ Nom. Plusieurs dénominations ont été proposées pour désigner la préfecture que la S.C. de la Propagande veut ériger: Préfecture d'Abbitibbi du nom de la principale mission sauvage. Préfecture de la Baie James, nom de la baie qui baigne ces provinces. Je préfère le nom de Préfecture de la Baie d'Hudson parce que ce nom mieux connu indique maintenant le point principal de la préfecture où sont réunis les principaux postes de la Compagnie. Géographiquement la dénomination n'est pas inexacte puisque la Baie James fait partie de la mer d'Hudson.

$2^{\circ}$ Limites. Il existe une difficulté pour délimiter exactement cette préfecture p.c.q. les limites actuelles entre les diocèses d'Ottawa et de $\mathrm{S}$. Boniface ne sont pas bien précises. Je propose donc de former la préfecture de la Baie d'Hudson, d'abord avec tous les districts du territoire de Rupers land qui sont situés entre la préfecture du Labrador et le diocèse de $\mathrm{S}$. Boniface; ensuite avec cette partie du territoire du Canada qui est arrosée par la Rivière 
Inclure une partie du territoire canadien

Titulaire
Ottawa et ses affluents jusqu'au confluent du Matawan à l'Ouest et de la rivière des Moines à l'Est. Peut-être, T.R.P. pouvons-nous prier la S.C. de décider la question des limites entre les diocèses d'Ottawa et de S. Boniface. Faut-il prendre pour limites une ligne géographique passant du cap Henriett et allant rejoindre les terres du Canada, Ce qui représente à peu près le $84^{\circ}$ Long $\mathrm{Gr}$, ou bien la ligne de démarcation doit-elle être faite de manière à comprendre dans le diocèse d'Ottawa toutes les contrées arrosées par les rivières qui envoient leurs eaux dans la Baie James en partant du cap Henriett. Si je propose d'attribuer à cette préfecture une partie du territoire canadien contrairement à ce que je propose pour la préfecture du Labrador, c'est que dans celle-ci $1^{\circ}$ Nos pères y ont été jusqu'ici seuls à exercer le st Ministère. $2^{\circ}$ Nos pères ne seront pas obligés d'y vivre isolément. $3^{\circ}$ Par l'œuvre des chantiers qu'ils soignent en hiver, ils peuvent se procurer quelques ressources. $4^{\circ}$ Les postes sauvages y sont multipliés quoique peu considérables. D'ailleurs je suis persuadé qu'avant peu d'années une partie de la préfecture en sera détachée pour former quelque nouveau diocèse.

$3^{\circ}$ Titulaires. Parmi les pères qui parlent les différents idiomes sauvages trois peuvent être présentés pour accomplir la haute fonction de préfet apostolique. Ce sont les RRPP XXX. Le conseil est unanimement en faveur du R.P. X d'autant plus que sa santé est meilleure. Ce serait fâcheux de prendre aussitôt le R.P. X qui est trop jeune. Quant au R.P. X il pourrait être préféré sous plusieurs rapports: il serait plus ingénieux, plus remuant que le $\mathrm{p}$. X mais il a un caractère difficile et je crains qu'il ne puisse vivre en bons rapports soit avec ses supérieurs, soit avec la compagnie de la B.H. Je dois ajouter qu'il ferait grandement défaut à la province.

Je crois R.P. avoir satisfait au devoir que vous avez voulu m'imposer. Permettez-moi de vous demander à mon tour quelques décisions pour régler certaines questions pratiques quand il faudra exécuter les décrets de la S.C. de la Propagande. Nos règles ne pourraient s'occuper des relations entre 
Quels

rapports

entre le

Préfet et

le Provin
cial? finances

pour les supérieurs locaux

une administration provinciale et celle de la préfecture. Permettez-moi de formuler en quelques questions. $1^{\circ}$ Quel pouvoir le provincial devra-t-il exercer dans les préfectures ? Aura-t-il le droit de visite ? Pourra-t-il déplacer les sujets dans les limites de la préfecture ou même en retirer les sujets ou les remplacer ? Devra-t-il contrôler les comptes de la préfecture ? Sera-ce le devoir du préfet de traiter directement avec NN SS les Evêques, soit avec les bureaux de la propagation de la foi ?

$2^{\circ}$ Dans quelle mesure les préfectures devrontelles contribuer aux frais généraux de la province?

$3^{\circ}$ Quelle position occuperont les préfets dans la province ? Seront-ils considérés comme supérieurs locaux et en dehors du préfet pourrait-il y avoir d'autres supérieurs locaux dans la préfecture ?

Veuillez, T.R.P., me permettre de vous faire acte de soumission en toutes choses. En obéissant à vos ordres j'ai exprimé librement ma façon de penser, mais avant tout nous voulons être soumis.

Montréal, 12 février 1871. Fl. VandenBerghe ${ }^{31}$.

Le 3 mars, le provincial écrivait au père Aubert:

Le jour de saint Joseph le nouvel archevêque de Québec sera consacré: nécessairement j'assisterai à la cérémonie et pendant que les évêques seront réunis, il y sera question des Préfectures Apostoliques, dont le poids retombera sur la Congrégation de toute leur pesanteur. Je suis encore irrésolu de ce qu'il convient de faire: m'appartient-il d'en parler avant que le décret du Saint-Siège soit connu et cependant l'occasion d'en parler est bien favorable ${ }^{32}$.

Quelques jours plus tard, c'est Mgr Guigues que le père Vandenberghe entretient du vicariat. Il veut ramener devant les évêques réunis à Québec la question de la préfecture, car Monseigneur de Montréal ne semble pas vouloir honorer sa signature. Mgr Guigues répond, le 7 mars; il estime étrange

31 Archives provinciales O.M.I., Correspondance, II : 244 et suiv.

32 Archives générales O.M.I., dossier Vandenberghe. 
qu'un évêque qui a pris l'engagement de contribuer pour la fondation de la nouvelle préfecture apostolique rétracte cet engagement ${ }^{33}$.

Le 10 mars, Mgr Guigues écrit de nouveau au père Vandenberghe:

Suivez vos bonnes inspirations de supérieur pour vous décider sur l'opportunité de parler ou de vous taire sur les engagements des Evêques par rapport aux préfectures. Si l'on a reçu de Rome quelque avis officiel sur cette nomination la règle de conduite est toute tracée; mais si l'on n'a rien reçu à Québec les délais et les changements qui ont eu lieu pourront faire regarder comme bien inopportune aux Evêques toute ouverture à ce sujet. Pour ce qui me concerne la résolution que vous prendrez sera la meilleure ${ }^{34}$.

Le 3 avril 1871, le père Aubert fait part au provincial de ses observations sur le mémoire relatif aux préfectures. Il ne veut pas entrer dans l'examen de toute la question; cela viendra plus tard, seulement il désire soumettre au provincial une difficulté qui lui semble très grave et primer tout dans cette affaire, bien qu'on la considère très secondaire. Il continue:

Quelles ressources peut-on attendre pour soutenir les deux préfectures? vous vous proposez de faire renouveler aux évêques de la province leurs engagements pour trois ans, et puis après que ferezvous ? Dans l'état où se trouve l'Europe et surtout la France peut-on compter sur l'œuvre de la propagation de la foi ? Il serait bon de faire entendre à nos Seigneurs canadiens que si nous ne refusons pas le travail nous ne voulons pas non plus nous exposer à l'odieux de nous retirer d'un poste de confiance et de dévouement, après l'avoir accepté, et cependant c'est le seul parti que je prévois pour nous, et je crois qu'il est difficile de prévoir autre chose, qu'on ne dise pas que dans des œuvres de cette nature que peut-on faire des calculs humains. Je crois au contraire qu'il faut les faire et que la Providence n'est pas tenue de faire un miracle pour nous procurer des ressources. Que les Evêques tiennent à nous

${ }^{33}$ Archives provinciales O.M.I., dossier Ottawa, 1ère maison, Evêché. 34 Ibidem. 
confier ces missions qui les délivrent du souci et de la responsabilité d'y pourvoir directement je le conçois, mais nous qui n'avons pas leurs ressources, nous devons encore plus tenir à ne pas nous en charger. Tâchez de faire comprendre cela aux évêques que vous verrez. Pour moi, soyez sûr que je ferai tout en mon pouvoir pour que l'affaire en reste là. Je manque peut-être de cet esprit d'abandon et de confiance qu'avaient les saints, mais je ne fais [que] ce que les lumières de ma raison qui elle-même est éclairée de Dieu, c'est pour cela qu'il nous la donne. Je ne sais comment nos pauvres missions qui ne vivaient que des aumônes de la propagation de la foi, vont faire dans les circonstances que les malheurs actuels nous ont faites ... ${ }^{35}$

La pensée du père Aubert est donc toujours la même: il ne faut pas accepter les préfectures et aller aux limites du possible pour les éviter. Le provincial écrit au général, le 23 avril 1871, et lui explique l'esprit de son rapport:

Il me semble que le Rév. Père Aubert se trompe complètement en regardant le rapport que je vous ai envoyé comme une instance pour obtenir l'érection des préfectures apostoliques en faveur de la Congrégation. Quand il s'est agi d'un Vicariat Apostolique le Conseil de la Province a donné son opinion bien motivée. Ensuite quand il fut question des préfectures, je crois vous avoir écrit que je regardais cette solution comme la plus malheureuse de toutes, car toute la charge des missions est laissée à la Congrégation et, de plus, elle crée une complication très difficile à l'administration provinciale. D'ailleurs le Père Aubert m'écrivit alors lui-même que ces préfectures étaient imposées à la Congrégation par Rome. Aussi j'ai fait mon rapport non pour examiner les avantages ou les inconvénients d'un refus ou d'une acceptation puisque la chose était jugée et que la Congrégation était obligée de se charger des Préfectures. Je n'ai voulu que répondre aux trois questions proposées par la Propagande et qui ont pour but l'organisation des préfectures. J'ai eu pour but d'obtenir des conditions aussi avantageuses que

85 Ibidem, dossier Administration générale. 
possible quant à la délimitation des préfectures. Je sais fort bien que l'entretien de ces préfectures est encore un problème que je ne puis résoudre et j'ai constaté que la Congrégation devra s'industrier pour trouver annuellement quatre ou cinq cents piastres. Si j'en crois donc la lettre du Père Aubert, il y aurait possibilité de modifier les décisions de la Propagande. Si c'est le cas, Très-Révérend Père, je vous prie d'user de toute votre influence pour que la Préfecture du Labrador ne nous soit pas laissée. J'ai eu l'occasion de voir l'évêque de Saint-Hyacinthe, qui parut tout étonné qu'il fût question des Oblats pour cette préfecture; il croyait qu'elle avait été donnée à des Franciscains. Quelle heureuse chose si cela était vrai. Quant à l'autre préfecture celle de la Baie d'Hudson ce serait peut-être possible et avantageux de l'accepter: 1) parce que l'Oeuvre des Chantiers nous donne des ressources; 2) parce que si la préfecture ne se fait pas, l'évêque d'Ottawa agira pour créer un évêché sur les limites ouest de son diocèse et par ce seul fait l'Oeuvre des Chantiers est enlevée à la Congrégation. C'est la raison qui l'empêche de négocier avec nous sur cette question. Voilà, Bien-Aimé Père, comment je comprends ces affaires. J'oubliais de dire, mais je l'ai constaté dans mon rapport, que les vues de l'archevêque de Québec avaient été d'annexer les côtes du Golfe de Saint-Laurent à un diocèse qui serait formé au Saguenay ${ }^{36}$.

Quelques jours plus tard, le provincial a l'occasion de rencontrer l'archevêque de Québec à Montréal, et lui parle des préfectures. Le nouvel archevêque semble partager les idées de son prédécesseur et a compris que la congrégation ne peut isoler ses sujets, surtout à des distances aussi considérables ${ }^{37}$.

L'évêque de Rimouski, si silencieux depuis assez longtemps, finit par rompre son mutisme. Le 6 mai, M. Langevin, secrétaire à Rimouski, annonce au provincial que le missionnaire de Natashquan revient à Rimouski et que l'évêque n'a personne pour le remplacer. D'un autre côté on s'attend à apprendre d'un moment

36 Archives générales O.M.I., dossier Vandenberghe.

37 A Fabre, 5 mai 1871 (ibidem). 
à l'autre qu'un des pères sera nommé préfet apostolique de la Côte-Nord. Dans ces circonstances, les Oblats pourraient peutêtre se charger immédiatement de Natashquan et des autres missions qui ne dépendent pas de la Pointe-des-Esquimaux ${ }^{38}$.

Le $13 \mathrm{mai}$, le provincial affirme au vicaire général de Rimouski, M. Langevin, ne pas savoir que penser au sujet de la préfecture de la Côte-Nord. Aucune nouvelle n'est encore arrivée de Rome et il a même entendu dire que la Propagande aurait eu l'intention de confier ces missions aux Franciscains. Même si la préfecture échoit aux Oblats, le père Vandenberghe ne voit pas comment le préfet pourra desservir les divers postes de la Côte, car la congrégation ne peut obliger ses sujets à vivre ainsi isolés. Force sera au préfet de trouver des prêtres de bonne volonté pour ce poste. Cette difficulté le préoccupe beaucoup et c'est pourquoi il n'ose prendre sur lui d'envoyer un Père à Natashquan pour y résider. Il espère cependant que la décision du SaintSiège sera bientôt connue et alors on ferait tout en son possible dans l'intérêt de ces pauvres missions dont la charge serait bien lourde dans les circonstances actuelles ${ }^{39}$.

On ne parlera plus de cette affaire avant le mois de septembre. On retarde donc toujours. Le 12 septembre, le père Aubert revient sur le projet, dans une lettre au provincial.

Vous savez que l'affaire des préfectures est toujours pendante et s'il dépend exclusivement de moi, elle le sera longtemps. Dernièrement le P. Corne procureur général à Rome m'écrivait que la Congrégation de la Propagande, réclamait les noms de ceux que nous devions proposer pour ces charges, je ne serais pas surpris si cette réclamation n'était pas urgée par quelques évêques du Canada. J'ai répondu au P. Corne que dans l'état présent nous croyions très inopportun la création de ces préfectures, parce qu'elles ne pourront être soutenues que par la ProRimouski.

39 Ibidem, Correspondance, II: 267-268 et archevêché de Rimouski, dossier Oblats. Sur le sujet de la vie isolée des missionnaires, voir Georgio Cosentino, O.M.I., La vie commune dans les missions étrangères selon notre Fondateur, dans Etudes Oblates, 15 (1956), 275-280. 
pagation de la Foi de Lyon qui dans l'état actuel ne peut se charger d'une pareille mission, d'autant plus qu'elle est brouillée avec les Evêques du Canada. Dans l'état présent les contrées qui doivent faire partie de ces préfectures dépendent des diocèses du Canada et les Evêques qui les possèdent sont tenus à les secourir, mais si ces contrées leur étaient enlevées, ils ne se croiraient plus tenus à les secourir, et toute la charge retomberait sur nous. J'espère qu'on n'urgera pas et qu'on se rendra à nos raisons dans l'intérêt même de ces missions. Je ne serais pas surpris qu'on vous en parlât en Canada, faites semblant d'être hostile au projet et laissez croire aux intéressés que vous devinez les motifs qui les portent à vouloir donner une solution à cette affaire, retranchezvous derrière l'impossibilité pour les temps actuels, d'être secouru par la propagation de la foi, nos guerres et les ruines qui en ont été la conséquence les aideront à comprendre cette impossibilité ${ }^{40}$.

On préférerait des procédés plus directs. Le père Vandenberghe répond, le 28 du mêrne mois, que l'archevêque de Québec s'est encore récemment informé des préfectures et que c'est avec lui que le provincial s'expliquera plus volontiers, car il n'insistera pas trop sur cette affaire puisqu'il presse présentement les Oblats à commencer un établissement sur le Saint-Maurice ${ }^{41}$.

A Rimouski, on ne dort pas. Le 29 septembre, deux lettres partent à l'adresse du cardinal Barnabo, l'une du vicaire général, M. Langevin, et l'autre de Mgr Langevin, évêque.

Dans la première on rappelle que Monseigneur l'évêque a déjà entretenu Son Éminence au sujet d'une préfecture pour les territoires situés à l'est et au sud de la Baie d'Hudson. Un mémoire à cet effet a été signé par tous les évêques de la province de Québec, présents à Rome l'année précédente, et soumis à la Congrégation de la Propagande.

Il est venu à la connaissance de l'évêque que plusieurs membres de la congrégation des Oblats, qui dans la vue des évêques devaient être chargés de la préfecture, hésitent à accepter vu les

40 Archives provinciales O.M.I., dossier Administration générale.

41 Archives générales O.M.I., dossier Vandenberghe. 
nombreuses missions dont la dite congrégation est chargée. Monseigneur de Rimouski espère toutefois qu'on pourrait triompher de l'obstacle vu que les Oblats ont déjà plusieurs missionnaires sur la Côte-Nord et que l'un d'eux, entr'autres, vient de faire une mission très importante dans l'intérieur; ils sont done parfaitement informés des mœurs des Indiens et même de leur langue. Le grand vicaire termine: "De plus ces estimables religieux ont un établissement important à Betsiamits qui pourrait servir de résidence principale ${ }^{42}$."

Mgr Langevin, de son côté, sollicite du cardinal Barnabo la permission de faire des instances pour que la Côte-Nord du fleuve Saint-Laurent soit détachée de son diocèse. Le quatrième concile provincial de Québec a déjà présenté une supplique en ce sens et, le 11 janvier 1869, l'évêque avait eu l'honneur d'exposer le projet en détail à Son Éminence. Le 27 avril de la même année, Son Éminence répond que la partie de la lettre qui a trait à cette affaire sera soumise à la congrégation en même temps que les actes et décrets du concile de Québec. Lors de son séjour à Rome pour le concile œcuménique avec les évêques de Trois-Rivières et d'Ottawa, un mémoire contenant toutes les informations désirées a été présenté à Son Éminence ${ }^{43}$.

Nous ne connaissons pas la réponse du cardinal.

De Paris, le père Aubert monte toujours la garde et donne des nouvelles au provincial, le 3 octobre 1871 .

Comme j'ai dû vous l'écrire déjà j'avais répondu au P. Corne qui nous pressait de nommer les préfets que nous désirions les voir choisis par la Propagande d'après bien entendu les instances de cette même propagande, que notre avis était que dans les circonstances actuelles, il fallait laisser les choses dans le statu quo et je développais les raisons qui nous portaient à suspendre l'exécution de ce projet. La congrégation a parfaitement compris nos raisons et comme nous elle comprend qu'il n'y a pas lieu pour le moment actuel surtout à créer les préfectures que

42 Archevêché de Rimouski, dossier Registre des Lettres, C, lettre 688.

13 Ibidem, lettre 689. 
les Evêques du Canada sollicitent. Si l'on vous en parle, j'entends les Evêques, répondez que la Propagande, dans l'état actuel de l'Europe, a suspendu l'exécution du projet et qu'il faut attendre des temps meilleurs. Je ne serais pas étonné que quelqu'un de ces prélats n'insiste pour qu'on donne suite au projet, je doute qu'il réussisse, dans tous les cas nous ferons notre possible pour maintenir le statu quo, qui je l'espère durera longtemps. Vous pouvez donc disposer de vos sujets sans tenir compte en aucune manière des deux préfectures ${ }^{44}$.

Le père Fabre écrit, de son côté, le 1er octobre, une lettre plus pacifique.

Nous avons obtenu que la création des fameuses préfectures apostoliques soit différée. Le P. Aubert a dû vous le dire, mais les Evêques du Canada ne vont-ils pas faire de nouvelles instances auprès de la Propagande ? c'est bien à craindre. Espérons cependant que cette création n'aura pas lieu de quelque temps ${ }^{45}$.

C'en est assez pour rassurer le père Vandenberghe. C'estlà, affirme-t-il au père Aubert, une "bien bonne nouvelle" ${ }^{46}$. Oui, le projet sera écarté pour longtemps, mais on continuera à y penser de temps en temps. Le 5 avril 1872, M. Langevin, vicaire général de Rimouski, écrit à Mgr Siméoni que les Oblats se rendent à la Baie-des-Esquimaux et ne savent à qui s'adresser pour la juridiction. Et il revient encore sur cette affaire de la préfecture ${ }^{47}$.

44 Archives provinciales O.M.I., dossier Administration générale.

45 Ibidem. Le projet était venu à deux doigts de réussir et, en 1871, sans que nous sachions la date précise, le provincial avait envoyé un mot à Mgr Guigues: "Je viens de recevoir de la part de notre père Général un avis qui nous fait présager que le décret apostolique pour ériger les préfectures des missions sera rendu prochainement. Je ne sais si je puis dans ces circonstances amener la question devant Nos Seigneurs les Evêques, qui réunis à Québec pourraient renouveler les engagements qui ont été pris à Toronto. Je le désire d'autant plus que l'évêque de Montréal ne paraît pas devoir faire honneur à sa signature. D'autre part nous ne pouvons pas compter sur les allocations des cuvres de Paris et de Lyon." (archives provinciales O.M.I., dossier Montréal, 1871). berghe).

46 Le 20 octobre 1871 (archives générales O.M.I., dossier Vanden-

47 Archevêché de Rimouski, Registre des Lettres, D, n 327. 
Le cardinal Barnabo, qui voit la lettre, écrit au père Fabre pour lui rappeler le projet conçu depuis quelque temps sur la formation d'une ou de deux préfectures dans le vaste territoire de la Baie d'Hudson. Le cardinal n'a pas oublié les difficultés qu'on lui avait exposées, mais il espère qu'on n'a pas cessé, pour cela, d'y penser et il espère que le général voudra bien faire en sorte que cette mesure puisse être adoptée le plus tôt possible ${ }^{48}$. Nous ne possédons pas la réponse du supérieur général.

Une fois la nouvelle connue au Canada que le projet des préfectures a été remis sine die, le père Flavien Durocher pense pouvoir exprimer clairement sa pensée au père Aubert. Il le fait longuement au mois d'août 1872.

Mais Grâces à Dieu et grand merci à nos Pères chargés des intérêts de notre Province, l'affaire de la Préfecture concernant ce territoire [le Saint-Laurent et le Labrador], a été remise et nous l'espérons à jamais pour le plus grand bien des âmes.

Vous connaissez, mon Révérend Père, tout ce qui a été allégué de notre part, pour empêcher cet objet. Voici quelques nouvelles données qui militent en notre faveur.

Le gouvernement canadien voulant établir des communications entre Québec sur le littoral nord du St Laurent a été obligé de mettre des bureaux de postes sur la rive Sud; et de la Gaspésie à l'extrême nord, il expédie la poste par voilier qui fait le service toutes les semaines, à Mingan, Pointe-aux-Esquimaux, et tous les 15 jours à Blanc-Sablon, limites de de notre Province de Québec. Si le courrier ne peut se rendre de la capitale de la province, par la côte nord du St Laurent, comment les Pères établis et résidants à la Rivière Moisie, à la Pointe-aux-Esquimaux, à Nataskouan (etc) pourraient-ils communiquer entre eux. Il faudrait donc deux Pères à chaque résidence pour desservir environ 45 à 100 familles par postes.

En outre le Gouvernement canadien vient de vendre à une Compagnie un township à 4 lieues au

${ }^{48}$ Le 2 mai (archives générales O.M.I., dossier Propagande). 
Nord de la baie de Betsiamits. Par là les sauvages de ce poste se trouvent circonscrits de toutes parts, environnés de Canadiens nombreux employés à l'exploitation du bois, des minérais, aux pêcheries, à l'agriculture. Avant peu, notre mission de Betsiamits, la plus florissante de toute la côte nord, deviendra semblable à celle du Lac des deux Montagnes. L'expérience est là. La mission de Mingan, autrefois la plus populeuse, est presque réduite à rien depuis l'établissement des Acadiens dans ces parages. La raison en est facile à comprendre, les blancs plus habiles à la pêche et mieux pourvus qu'eux accaparent tout.

Avant peu d'années la tribu montagnaise sera refoulée dans l'intérieur des terres et si nos Pères s'établissent définitivement à l'extrémité ouest de la Grande Baie des Esquimaux au confluent des rivières Naskapie et Hamilton, comme il en est question, les montagnais se rendront à leur résidence, abandonnant le littoral du St Laurent.

Ce que nous désirons c'est qu'on laisse les choses dans l'état actuel où elles sont.

Mgr de S. Germain de Rimouski, qui veut faire de nouvelles instances pour se débarrasser des résidences de la côte nord du St Laurent, peut néanmoins visiter ces lieux assez facilement. De sa résidence aux Betsiamits, il n'y a qu'une quinzaine de lieues à faire par eau; puis de la Gaspésie, incluse dans son diocèse, à l'extrémité est de la côte du nord s'y rendre en deux jours par le voilier de la malle.

D'un autre côté, la partie du Labrador appartenant à Mgr du Havre de Grâce, Isle de Terre-Neuve, continuerait à être visitée par les Prêtres de ce dernier diocèse. L'évêque du Havre de Grâce peut facilement pourvoir au bien spirituel de ses diocésains établis au Labrador. Je ne sache pas qu'il demande à en être déchargé.

Il est facile de concevoir quel fardeau incomberait à notre Congrégation, si elle était chargée de la préfecture en question, et les résultats en seraient très minimes. En nous occupant uniquement des Sauvages nous pourrons nous avancer vers Moose 
[Baie] et enlever aux Moraves et aux protestants méthodistes une partie des sauvages qui désirent la visite de nos missionnaires.

Vous savez sans doute que la Compagnie d'Hudson nous favoriserait dans le projet d'établir une résidence à la Baie des Esquimaux. Le Gouverneur de cette Compagnie l'Honorable Smith s'est engagé, par écrit, à payer une annuité aux Missionnaires ${ }^{40}$.

Le provincial jouira de la paix jusqu'en 1875 . A ce moment, l'évêque de Rimouski lui écrit, le 13 août, au retour de sa visite de la Côte-Nord. Il insistait de nouveau, auprès des Oblats, les suppliant, pour le bien spirituel de la Côte-Nord, de se charger de tout ce territoire sous la direction d'un préfet apostolique, résidant au milieu de la population. Les prêtres séculiers pourraient rester dans la préfecture jusqu'à ce que la Congrégation ait assez de prêtres pour les remplacer ${ }^{50}$. Le 20 août, le provincial répond qu'il fera part de son désir au supérieur général ${ }^{51}$.

Nous ne savons pas si le père J.-E. Antoine, provincial, en écrivit au supérieur général, mais le 25 octobre, il écrit au père Arnaud probablement:

Mgr de Rimouski fait de nouvelles instances pour se débarrasser de toute la côte nord en faveur des Oblats; si nous étions en nombre, je serais de suite de son avis ${ }^{52}$.

La question est continuellement agitée, il est difficile de l'oublier. Le 13 mai 1876, le père Tortel s'adresse au père Aubert dans une longue lettre; il y refait toute l'histoire de cette question.

D'abord la question du Vicariat Apostolique du Labrador et de la côte Nord.

49 Ibidem, dossier Durocher, Flavien.

50 Archives provinciales O.M.I., Correspondance, II : 421 et archevêché de Rimouski, Registre des Lettres, F.

Au chapitre général de 1873, Mgr Paul Durieu, vicaire apostolique de la Colombie-Britannique, avait fait voter une proposition à l'effet de n'accepter aucun nouveau vicariat apostolique dans la congrégation aussi longtemps que les vicariats dont elle était chargée ne seraient pas pourvus du personnel suffisant (p. 159).

51 Archives provinciales O.M.I., Correspondance, II : 425.

52 Ibidem, dossier Betsiamites. 
Cette question a déjà été étudiée par le Conseil Provincial. En janvier 1868 la délibération au sujet d'un Vicariat Apostolique au Labrador se formulait ainsi: "Tous reconnaissent à l'acceptation de ce Vicariat des inconvénients graves et des difficultés réelles. C'est multiplier dans la Congrégation des divisions administratives pour une population qui n'excède pas cinq mille âmes. Le Vicaire de ces missions gênera l'administration Provinciale parce qu'il sera souvent dans le Canada, si même il n'est pas obligé d'y avoir sa résidence supposant que l'on conserve les limites indiquées par la lettre des Evêques. L'on prévoit aussi de très-graves difficultés quant aux ressources. Les Evêques ont fait une souscription de trois ans seulement, ensuite le Vicaire devra tout attendre du Bureau de la Propagation de la Foi de France et de la charité publique. Il est fort douteux que la Propagation de la Foi consente à prendre sur elle les charges des Evêques du Canada. C'est donc un état tout-à-fait précaire qui n'a rien d'assuré."

Par une lettre du 10 mai 1870, le Rév. Père Aubert annonce officiellement "que la S. Propagande de Rome veut confier à notre Congrégation deux Préfectures Apostoliques; l'une à l'Est, l'autre à l'Ouest du Canada. Notre Très-Rév. Père Général a décidé que ces Préfectures dépendraient immédiatement de la Province du Canada sous le rapport religieux. Le Cardinal Barnabo veut que le TrèsRévérend Père Général désigne lui-même le nom de ces Préfectures, les limites à assigner et les sujets qui seront titulaires. Le conseil n'a traité cette fois qu'une seule question, celle des limites. Pour la Préfecture de l'Est, la question est de savoir si avec le Labrador la Préfecture doit comprendre aussi les côtes du Golfe. Dans cette supposition, il nous faut trouver quatre prêtres de plus au moins, et chercher les moyens de les entretenir; or, plus que jamais les moyens d'entretien sont incertains et nous n'avons pas un nombre suffisant de sujets. Nous ne pouvons compter sur la Propagation de la foi, le concours des Evêques n'est promis que pour trois ans et encore l'Evêque de Montréal a-t-il déjà retiré la souscription faite en 1867. Il est reconnu aussi que les 
prêtres actuellement fixés sur les côtes, sont à la charge de l'évêque de Rimouski. Après un examen sérieux, il fut décidé que l'on tenterait tous les efforts pour que la Préfecture fût restreinte à Labrador, avec exclusion des côtes du Golfe." Par cet extrait, vous avez un aperçu historique qui montre la répugnance motivée, qu'éprouvait l'Administration provinciale, soit pour un Vicariat soit pour une Préfecture. Aujourd'hui le Conseil entend dire que la question loin d'être enterrée est plus vivante que jamais. Monseigneur Langevin, évêque de Rimouski, fait son cauchemar de la côte Nord, il a écrit lettres sur lettres à Rome. Monseigneur Taschereau, archevêque de Québec, opine d'accord avec son suffragant qui lui doit cette portion ardue de son champ, les autres Evêques suffragants ne peuvent que se montrer courtois à l'égard de leur collègue qu'il s'agit d'exonérer d'une lourde charge. Le Conseil inquiet de ces dires croit de son côté devoir revenir à la charge et supplier l'Administration générale de vouloir bien peser ses raisons et l'appuyer de son refus. La question n'est plus, paraît-il, celle du Labrador que Monseigneur de Rimouski trouve tout à fait onéreuse à soigner soit par lui-même dans la visite Pastorale soit par ses Prêtres qui refusent les trois ou quatre postes où se trouvent agglomérés les blancs échelonnés le long de la côte. On trouve tout simple et très commode de s'agiter pour s'en débarrasser et en obérer la Congrégation qui se trouverait ainsi obligée de pourvoir à ces trois ou quatre résidences fournir le Vicaire de plusieurs autres sujets formant un état major indispensable et cela sans aucune ressource assurée et permanente. Le secours offert par les Evêques pour trois ans ne saurait allécher et n'est pas sérieux. Pour tous ces motifs, c'est-àdire, le défaut de sujets, le défaut de ressources, le danger constant de perdre les sujets que l'on aurait à employer isolément et qui ne peuvent y être forcés. Toutes ces raisons semblent graves et majeures et font insister pour que les Evêques nous fassent grâce de cette nouvelle fondation. Jusqu'à présent la Province du Canada a donné tous les secours en son pouvoir aux sauvages errants de la côte Nord; elle est toujours disposée avec l'aide de Dieu à continuer cette œuvre et à la développer avec bonne volonté et 
dévouement sous la juridiction épiscopale, mais elle ne voit pas possibilité à prendre la charge des blancs qui occupent ce territoire. Le nouveau diocèse que l'on veut fonder à Chicoutimi dans le Saguenay pourrait très-bien faire face à cette obligation et nous épargner au moins une source inépuisable de difficultés toutes plus ardues les unes que les autres. Malgré l'insistance des Evêques, le Conseil Provincial espère encore qu'il sera fait droit à ses réclamations et il se trouve encouragé dans cet espoir par une parole de Monseigneur Bourget qui nous dit que la S. Propagande ne nous obligeait pas. Veuillez saisir le Conseil au plus tôt de cette affaire et diriger vos batteries en conséquence et Dieu et son Immaculée Mère nous préservent de cette calamité ${ }^{53}$.

Le Conseil général, pour sa part, étudie encore le problème, le 25 juin. Sa réponse est négative. Le père Antoine, affirme-t-il, tient entre ses mains les raisons du refus ${ }^{54}$. Le 10 octobre, les membres du Conseil notent que le père Antoine, provincial, se propose de voir Mgr Conroy, délégué apostolique, pour lui faire agréer ses raisons ${ }^{55}$.

Le 8 août 1876, le père Arnaud écrit au père Soullier. Monseigneur de Rimouski, lui confie-t-il, ne parle plus de préfecture et le père ajoute: "il pourrait facilement se débarrasser de nous en donnant toute la côte au nouvel évêque de Chicoutimi ${ }^{56}$."

Le 8 septembre, le Conseil général note de nouveau que Monseigneur de Rimouski veut imposer aux Oblats le nouveau vicariat du Labrador. Si l'on accepte, disait-on, les évêques seront obligés de soutenir financièrement les missionnaires. Or comme ils se désintéressent et que les sujets ne sont pas assez nombreux, nous ne pouvons accepter les offres d'un évêque trop intéressé.

Le projet renaîtra en 1877. Au mois de juin, le Père Antoine nous apprend qu'à la fin de mai Mgr Conroy, délégué apostolique

53 Archives générales O.M.I., dossier Tortel, Adolphe. Voir aussi les Minutes du Conseil provincial, II: 224.

54 Page 268. Voir aussi la séance du conseil général, le 7 juillet 1876.

55 Page 280.

56 Archives générales O.M.I., dossier Arnaud, Charles. 
au Canada, fait sa première visite à Saint-Pierre de Montréal et que déjà Mgr Langevin, de Rimouski, a saisi Son Excellence de la question du vicariat resté jusqu'alors à l'état de projet. Mais le provincial a fait remarquer au délégué apostolique que le supérieur général avait déjà refusé le vicariat et que la Sacrée Congrégation avait accepté ses raisons; lesquelles raisons existaient toujours et la congrégation ne manquerait pas de les faire valoir. A la demande du délégué apostolique, le provincial rédige le rapport suivant, en date du 5 juin 1877. Nous en donnons le contenu:

La pénurie de missionnaires et le manque de ressources empêchent les Oblats de se charger de cette œuvre. Il y faudrait de huit à dix missionnaires et ce n'est qu'avec un effort suprême qu'on a réussi à porter ce nombre à quatre. Les évêques ont consenti à faire une souscription d'environ $\$ 2,000$, mais pour trois ans seulement. Les missionnaires ne peuvent vivre dans l'isolement; puis quatre postes sont desservis par des prêtres séculiers; il est impossible à l'Ordre de remplacer ces prêtres. Cependant les Oblats acceptent de continuer la desserte des Indiens et de les visiter aussi souvent que possible. En outre, la Congrégation de la Propagande ne peut oublier que les Oblats ont fait des efforts presque surhumains pour maintenir cinq vicariats apostoliques en Amérique du Nord ${ }^{57}$.

Le Conseil général délibère sur le rapport du Père Antoine le 25 juin. Le Conseil refusait le vicariat, se fondant sur le mémoire du père Antoine ${ }^{58}$. Le lendemain, 26 juin, le Père Aubert fait savoir de Paris que le Père général ne peut que maintenir sa décision par rapport au vicariat apostolique de la CôteNord ${ }^{59}$. Le 28 juin, le père Fabre affirmait au père Antoine: l'on ne veut du vicariat à aucun prix ${ }^{60}$.

Les Oblats se verront libérés de la préfecture par suite de leur refus constant de s'en rendre responsables. Le 2 août 1877,

57 Archives provinciales O.M.I., Correspondance, III: 11-13.

58 III : 268.

$59 \mathrm{Au}$ père Antoine (ibidem, III: 14).

60 Ibidem, 15. 
le père Antoine écrit, probablement au père Soullier, qu'il descendait à Rimouski pour la visite pastorale; il sera encore question du vicariat de la Côte-Nord, prévoit-il; mais il se dit préparé à la guerre qu'il devra soutenir à ce sujet avec Mgr Langevin... "mais je me propose de demander à Sa Grandeur d'attendre la fin de la retraite pour commencer les hostilités, ce sera le moyen de les terminer bien vite... ${ }^{61}$ "

Nous voyons par un rapport du Conseil général tenu le 10 août 1877 que le père Antoine se propose de voir Mgr Conroy, délégué apostolique au Canada; il entend lui faire agréer les raisons des Oblats quant au refus du vicariat apostolique du Labrador ${ }^{62}$.

Cette démarche apportera le résultat désiré; les évêques jetteront les yeux sur un prêtre séculier. Le Père Antoine annonçait au père Fabre, le 2 août 1878, qu'en passant à Rimouski, il a vu le prêtre choisi par les évêques pour la préfecture apostolique du Labrador. Il s'agissait du curé de la cathédrale, M. Auger, qui cherchait à décliner l'honneur. Le père Antoine affirme que le curé réussira si le nouvel évêque de Chicoutimi (Mgr Dominique Racine) veut se charger de toute la Côte-Nord ${ }^{63}$. A ce moment il semble que l'évêque de Rimouski a perdu tout espoir, car le père Arnaud écrit au père Fabre, le 30 décembre 1878: "Mgr de Rimouski a perdu l'espoir d'ériger notre littoral en préfecture apostolique. Il pourrait le donner au nouvel évêque de Chicoutimi, sacré le 4 août ${ }^{64}$." A cette époque, Mgr Langevin adresse une supplique au Saint-Siège à peu près en ces termes: "Puisque les Oblats ne veulent pas se charger de la Préfecture Apostolique du Labrador; j'avais proposé le nom du R.P. Antoine, provincial, comme vicaire apostolique, je soumets l'autre nom de la première demande, M. Joseph Julien Auger ${ }^{65}$."

61 Archives générales O.M.I., dossier Antoine, Joseph-Eugène.

62 III : 280.

63 Archives générales O.M.I., dossier Antoine.

64 Ibidem, dossier Arnaud.

65 Evêché du Golfe Saint-Laurent. Le 28 octobre 1878, le Conseil général refusait d'accepter la nomination d'un Oblat au vicariat apostolique de Pontiac afin qu'on ne revînt pas sur l'affaire du Labrador. 
En fait, la préfecture apostolique du Golfe Saint-Laurent ne sera fondée que le 15 juin $1882^{66}$ et Mgr François Bossé en sera le premier titulaire. L'avis de sa nomination est daté de Rome, du 28 juillet 1882 et, en 1892, Mgr Bossé se réjouit de voir la préfecture passer sous la juridiction de Chicoutimi ${ }^{67}$.

Le 26 juin 1884, Mgr Bossé, dans une lettre à Mgr Lorrain, vicaire apostolique de Pontiac, lui apprend qu'au cours de l'hiver, Mgr McDonald, de Terre-Neuve, a demandé aux Oblats de Betsiamites d'évangéliser la côte est du Labrador. Mgr Bossé a même payé, dès octobre 1883, les Pères pour évangéliser la partie nord; on le lui a permis. Mais au printemps, le père Lacasse, sur qui Mgr Bossé comptait, prenait une autre direction. Les deux autres Pères, Babel et Arnaud, étaient trop vieux pour ce voyage et ne songeaient même pas à l'entreprendre.

Mgr Bossé possédait les renseignements suivants: les Nascapis vivaient dans l'intérieur au nombre d'environ cinquante familles et diminuaient très vite. Les Esquimaux comptaient de deux cents à deux cent cinquante familles et diminuaient aussi, mais moins rapidement. Les dépenses étaient telles qu'il était difficile de fonder une résidence. Ungava, cependant, semblait un lieu central à l'est et à l'ouest. Quant à lui, il serait prêt à donner de cinq à six cents dollars par année, pris sur la quête du Nord-Ouest.

Mgr Bossé parlait aussi de fondations sur la côte est et à la Baie James et de préfecture à Blanc Sablon et à Moose Factory, sur la côte Nord et au lac Saint-Jean, mais sans juridiction sur la population blanche. Il disait à Mgr Lorrain qu'il pouvait en entretenir le délégué apostolique.

Le Père Arnaud avait assuré Mgr Bossé de voir en tout cela un bon plan; et maintenant il apprenait que les Oblats estimaient son plan irréalisable; il en coûterait selon eux des sommes fabuleuses pour entretenir des prêtres résidents en ces régions. Mgr Bossé avait assez fait preuve de bonne volonté, prétendait-il. Il

${ }^{66}$ Gaston CARRIÈRe, O.M.I., San Germano di Rimouski, dans Enciclopedia Cattolica, X: col. 1766-1767.

67 Evêché du Golfe Saint-Laurent. 
fallait cependant les Oblats dans ces régions, car une communauté religieuse remplacerait plus facilement les missionnaires fatigués qu'un supérieur ne possédant même point un grand séminaire. Quant à la position des Indiens, elle était semblable à celle de ceux de l'Athabaska, où les Oblats étaient établis.

En 1891, Mgr Bossé croit encore en la possibilité de faire passer la préfecture aux Oblats. Il écrit à Mgr Bégin:

Nos Sœurs arrivant de Québec me rapportent qu'il y a rumeur accréditée que les nouvelles nominations pour la Coadjutorerie et Chicoutimi sont faites et seront publiées à la Retraite - que cette Préfecture sera confiée aux Oblats à être desservie comme Mission, que je serai mis curé, et qu'une nouvelle préfecture sera formée dans la Gaspésie et Baie des Chaleurs. ... Je sais que Mgr de Sherbrooke était d'opinion il y a deux ans que la Préfecture devait être confiée à un ordre religieux, pour assurer des prêtres au besoin ${ }^{68}$.

Mgr Bossé veut cependant persévérer encore un an. Mgr Langevin, dit-il, en fin de sa lettre, avait cette étrange opinion que le préfet apostolique "une fois installé se créerait ses propres ressources. Créer des ressources pour soutenir un clergé, écoles et chapelles, même créer des prêtres à même les roches du Labrador et un très pauvre peuple de pêcheurs !...69" La préfecture n'avait donc pas donné tous les fruits que l'on en attendait avant sa création. Les Oblats auraient-ils pu faire mieux, dans les circonstances? Il n'est pas facile de le savoir.

Un dernier effort sera tenté en 1901. Mgr M.-T. Labrecque de Chicoutimi s'adresse à Mgr Nazaire Bégin, à Québec, le 28 septembre 1901. Il lui fait part d'un projet très important relatif à la Préfecture du Golfe ... il n'est plus capable d'y envoyer des prêtres; et il ne sait à qui remettre ce territoire:

J'ai pensé aux Oblats. Qu'en pense Votre Grandeur ? Je crois qu'ils se chargeraient volontiers de la Préfecture dans les conditions actuelles. Tout

68 Evêché du Golfe Saint-Laurent.

69 Ibidem. 
est bien organisé, les revenus de chaque mission sont assez bons pour le missionnaire et la fabrique; un capital de $\$ 20.000$ à leur service; $\$ 400$ piastres d'allocation annuellement pour les écoles et une dette seulement de $\$ 2000$ contractée par Mgr Bossé lors de la construction du couvent de la Pointe.

Mgr Labrecque dit, en outre, posséder un moyen de remettre l'argent du capital et donc il serait ainsi débarrassé de "cet éléphant":

Si votre avis s'accorde avec mes vues sur ce sujet, je pourrais dès maintenant correspondre avec le Père Provincial des Oblats pour m'assurer s'ils accepteront la Préfecture dans le cas où Rome la leur confierait. S'ils refusent, il sera facile de trouver des Religieux en France à l'heure qu'il est ${ }^{70}$.

Nous ne savons pas quelle fut la réponse de Mgr Bégin, toutefois la préfecture ne passa point aux Oblats. Et voilà qui termine cette question de la préfecture du Labrador. Avait-on oublié celle de la Baie d'Hudson ? Certes non. En 1878, Mgr Duhamel projette de faire ériger le vicariat apostolique de Pontiac. Le Père Antoine a échappé à la charge de préfet apostolique de la Côte-Nord. Le voici maintenant menacé de celle de vicaire apostolique de Pontiac.

On lit, en effet, dans les minutes du Conseil général, le 13 septembre 1878, que Mgr Duhamel demande à se faire accompagner à Rome par le père Antoine. Il s'agit sans doute de l'Université, mais on soupçonne que Mgr Duhamel veut un oblat, et peut-être le père Antoine, pour le nouveau diocèse ${ }^{71}$. Le 18 octobre 1878, le rapport du Conseil nous informe que Mgr Duhamel veut faire nommer un Oblat pour l'administration de la partie nord de son diocèse. Le Conseil se rassure cependant, car Rome ne fera rien sans consulter la congrégation ${ }^{72}$. Cette nouvelle, dit le rapport du Conseil, est venue du père Tortel. Il avait, en effet, écrit à ce sujet le 27 septembre $1878^{73}$ et, le 28 octobre, le Conseil s'intéresse encore à cette affaire. Mgr Duhamel, y

70 Archevêché de Québec, dossier D.C. 1-144.

71 III: 349.

72 Ibidem, 356.

73 Archives générales O.M.I., dossier Tortel. 
est-il dit, veut diviser son diocèse et $\mathrm{y}$ faire nommer un Oblat, en l'occurrence, le Père Antoine. Ce père refusera pour la congrégation et pour lui. La partie à détacher du diocèse d'Ottawa n'est pas entièrement "sauvage"; elle comprend une douzaine de paroisses. L'évêque affirme que, par la division, il perdra un tiers de son revenu. Les Oblats continueront à s'y dépenser, mais sans un nombre de sujets suffisant. Si ce clergé est moitié religieux et moitié séculier, il vaut mieux que l'évêque soit séculier. Enfin, si l'on accepte, il y a risque qu'on revienne sur la question du Labrador ${ }^{74}$.

Mgr Duhamel, de passage à Paris, rend visite au Père Antoine. On s'entend sur tous les points relatifs à l'Université et on est décidé à tout faire pour appuyer les desseins de l'évêque relativement à la division de son diocèse, mais le supérieur général est opposé à la nomination d'un Oblat. Eût-il connu les motifs de ce refus, Monseigneur a-t-il semblé dire, n'aurait pas commencé les démarches ${ }^{75}$. Le Père Antoine, de son côté, reste inquiet. Pourra-t-il décliner l'épiscopat? Une lettre envoyée de Rome ne se fait pas très rassurante ${ }^{76}$.

Le 30 décembre 1878, on lit dans les minutes du Conseil:

Lecture est donnée d'une pièce de la Propagande et accompagnée d'une lettre du card. Simeoni au T.R.P. Supérieur Général. L'une et l'autre ont pour objet d'inviter le Supérieur Général à consentir à la nomination d'un Oblat au nouvel évêché de Pontiac, sur l'avis qu'il avait reçu de Mgr Duhamel, se propose d'écrire de nouveau, en réponse à cette nouvelle et officielle communication, pour renouveler l'expression de son refus et de ses regrets. Il se fera appuyer par le card. Guibert ${ }^{77}$.

Le 14 janvier 1879, le Père Antoine écrit au père Fabre: Mgr Duhamel tient de la part du cardinal préfet la réponse du supérieur général relativement au vicariat apostolique ${ }^{78}$. Enfin,

74 III : $357-358$.

75 Ibidem, 359.

76 Ibidem, 364 (16 décembre 1878).

77 Ibidem, 366.

78 Archives générales O.M.I., dossier Antoine. 
le supérieur général a obtenu gain de cause. Une lettre de Mgr Duhamel lue à la réunion du conseil général, le 27 janvier 1879, confirme la nouvelle au sujet du vicariat de Pontiac. Une lettre du cardinal Guibert, O.M.I., a aidé à cette décision ${ }^{79}$. Le vicariat sera érigé le 11 juillet 1882 et Mgr Lorrain, nommé premier vicaire apostolique.

Ainsi se termine l'affaire des vicariats et des préfectures. Le Père Antoine, proposé à la préfecture du Labrador et à celle de vicaire apostolique à Pontiac, a réussi à éviter la mître. Son nom avait été aussi mentionné dans la terna préparée par Mgr Guigues pour la succession à Ottawa ${ }^{80}$.

Les Oblats avaient donc réussi à éviter la mître. On avait retardé, du même coup, l'érection de préfectures apostoliques spéciales pour les régions de la Baie James et du Labrador. Les préfectures auraient pu accomplir, sans nul doute, un grand bien pour l'extension de la foi catholique. Mais le temps était-il vraiment venu ? Il n'est pas facile de répondre à cette question. Il ne fait pas de doute cependant, qu'en dépit de l'opposition, peutêtre systématique du père Aubert, les difficultés indiquées par lui ne manquaient point de fondement. Dans les circonstances des préfets apostoliques oblats auraient certainement eu beaucoup de difficultés à développer l'Église, tant à cause du manque d'ouvriers apostoliques que du peu de secours financiers sur lesquels ils pouvaient compter.

GASTON CARRIÈRE, o.m.i.,

Institut d'Histoire, Université d'Ottawa.

79 IV : 5 .

80 Antoine à Fabre, 26 mars 1874 (archives générales O.M.I., dossier Antoine). 